\title{
TWO CARDIAC CASES SHOWN TO THE M.R.C.P. CLASS.
}

\author{
BY TERENCE EAST, M.D., \\ Physician, King's College Hospital and Woolwich War Memorial Hospital.
}

THE first case shown was an elderly man with syphilitic aortitis and some dilatation of the aorta. The history of the symptoms was suggestive. A man, aged 57, who had performed heavy manual labour, had found that during the last few months he had suffered from dull precordial pain on exertion, and increasing shortness of breath.

Physical examination showed a man of medium build. The pulse was regular, with a rate of 80 . The tension moderate. The wave fairly good; neither definitely water-hammer nor collapsing. The brachials and radials were rather thickened and tortuous. The chest showed a good deal of emphysema, no doubt partly due to his occupation, which was that of a coal-heaver.

These changes in the lungs made detection of the signs in the heart more difficult. Examination of the precordial area showed that there was slight increase in dullness over the upper part of the sternum. There was definite strong upward pulsation in the suprasternal notch; slight pulsation over the upper part of the sternum could be seen. The apex beat was difficult to locate, but seemed a little further to the left than normal. On auscultation, the sounds at the apex were less distinct than usual, but clear.

At the base there was a short systolic murmur on the aortic area, rather coarse in type, conducted a little way upwards, and low in pitch.

The aortic second sound was hollow and rather musical, with some increase in? intensity and heard over a large area upwards. Down the left of the sternum was a short, soft, diastolic murmur, conducted a little way downwards. The liver and jugular veins were normal.

Instrumental investigation showed that the B.P. was $156 /$ I04.

The skiagram showed considerable irregular increase in the size of the aorta in all its extent in the thorax ; most noticeable in the ascending part.

The heart shadow was enlarged, mainly in the left ventricle.

The electrocardiogram showed preponderance of the left ventricle with flattened T. waves. Candidates made mistakes mainly on the following points :-

The dilatation of the aorta was missed because the significance of the hollow musical aortic second sound was not realized. The importance of this sign was all the greater since the blood-pressure was not very high. This abnormal type of aortic second sound can be differentiated from the higher pitched more localized accentuation due to high blood-pressure alone.

Pulsation was not always felt for in the suprasternal notch, nor the pulsation over the manubrium seen. The slight dullness over the upper part of the sternum was overlooked because candidates omitted to take into account the obvious degree of emphysema. For the same reason the enlargement of the left ventricle was missed. In these cases careful examination in full expiration is most important.

The aortic leak was overlooked because the diastolic murmur caused by it was rather low on the left side of the sternum; also the absence of a characteristic pulse of aortic reflux rather misled candidates. 
The skiagram was usually commented on correctly, but some were very vague about the nature of various contours of the heart shadow. Most candidates recognized the electrocardiogram.

The second case was a middle-aged man with a moderate degree of mitral stenosis and auricular fibrillation. Here the ventricular rate was well controlled by digitalis. In consequence the arrhythmia was misdiagnosed fairly often, some thinking that extra-systoles only were present. Sometimes with slow ventricular rate the irregularity is slight, but that there is never true regularity should always be recognizable on car eful observation. Acceleration of the heart rate will always make the irregularity more obvious. The mitral lesion was not always correctly assessed. Owing to the presence of auriculation fibrillation only the murmur in the earlier part of diastole was heard. Not many candidates took advantage of the procedure of turning the patient on the left side in order to increase the loudness of the murmur.

In this case the degree of precordial thrust over the right ventricle was not always correctly appreciated.

The associated recession of the intercostal spaces just outside the left border of the heart was mistaken for systolic retraction and an adherent pericardium diagnosed.

The electrocardiogram was for the most part correctly interpreted.

These two cases should have presented no real difficulties if accurate examination and investigation of the physical signs had been carried out.

\title{
A CASE OF DIABETES WITH CHRONIC PULMONARY TUBERCULOSIS AND SYPHILITIC AORTITIS.
}

\author{
By PHILIP ELLMAN, M.D., M.R.C.P., \\ Physician-in-Charge of Tuberculosis Dept. and Chest Clinic, East Ham.
}

THIS case was shown by me to the M.R.C.P. candidates primarily as a chest case. In order to assist candidates with the limited time at their disposal, I told them that the patient had diabetes and a positive Wassermann. With few exceptions I found that the candidates diagnosed either pulmonary tuberculosis or syphilitic aortitis, but not both.' The patient was a man in the 50's, with a very suggestive history of chronic chest disease. I think the reason why the complete diagnosis was missed was because of the faulty method of examining chest cases ; most candidates proceeded at once to examine the lungs. It is essential in all chest cases to make an examination of the heart first, especially with regard to its size and position. Had this been done one feels certain that the aortitis would not have been missed. It must be admitted that the physical signs of the aortitis were few, although the characteristic ringing aortic second sound was beyond question and was the clue to the diagnosis. With regard to the lungs, most candidates had diagnosed the lesion in the right upper zone as a fibroid lung. This of

\footnotetext{
1 In addition the patient was a tabetic with Charcot's knees.
} 